\title{
Ulcerative oesophagitis in association with ulcerative colitis
}

\author{
CLIVE ROSENDORFF AND N. W. T. GRIEVE \\ From the Departments of Medicine, St. Thomas's Hospital Medical School, and Radiodiagnosis, \\ St. Thomas's Hospital, London
}

\section{EDITORIAL COMMENT A case report describing a possible new complication of proctocolitis.}

Ulcerative colitis is primarily a disease of the large bowel, but a wide variety of extracolonic complications of the disease are now recognized (Edwards and Truelove, 1964), and a number of these occur in other parts of the alimentary tract.

In the mouth, aphthous ulcers and monilial infections arise as a result of the general debility caused by the colitis (Grieve, 1964). Gastritis and small intestinal changes were found in a high proportion of patients with ulcerative colitis by Salem, Truelove, and Richards (1964), and are of more uncertain aetiology. It is possible that immunological factors, such as those described by Taylor (1965a and b), may play a part.

In 1956, Berdal and Harnaes described two patients in whom dysphagia occurred during the course of a severe attack of ulcerative colitis. Both were found to have multiple ulcerations of the oesophagus, which eventually improved, leaving fibrous strictures.

This paper records a similar occurrence in a young man suffering from ulcerative colitis. We can find no previous account of this complication in the British literature.

\section{CASE HISTORY}

A.P.C., a 23-year-old white male, was admitted to St. Thomas's Hospital on 3 January 1965. His complaints were of intermittent joint pain and fever for the previous seven years; of episodes of diarrhoea, latterly with blood and mucus in the stools, for six years; and of dysphagia of increasing severity for two years.

At his first hospital admission, at Ashford, Kent, in 1958, when fever, arthralgia, and tachycardia were the features of his illness, a diagnosis of acute rheumatic fever was made. He was severely ill and steroids were used as part of his treatment. Thereafter tonsillectomy was performed and a prophylactic course of oral penicillin

1 Present address:

Department of Radiology, St. Peter's Hospital, started. The following year, he was again admitted to hospital and on this occasion diarrhoea and buccal ulceration were present. In 1960, during a further episode of diarrhoea, a diagnosis of ulcerative colitis was made on the basis of sigmoidoscopy and barium enema examination.

Dysphagia appeared for the first time in 1963, and a barium swallow at that time showed abnormality of the oesophagus suggestive of monilial infection. Over the next two years, the dysphagia became progressively more troublesome.

Since 1959 he had been virtually continuously on maintenance steroid therapy, with temporary increases during the exacerbations of the illness.

On examination he was not anaemic and there were no cutaneous lesions. The pharynx was mildly hyperaemic, and examination of the abdomen revealed no abnormalities. The cardiovascular system was normal and there were no abnormalities in the respiratory or central nervous systems.

Haemoglobin was $12.9 \mathrm{~g}$. per $100 \mathrm{ml}$., serum iron level $60 \mu$ g. per $100 \mathrm{ml}$., white blood count $8,250 / \mathrm{c} . \mathrm{mm}$. (neurophils $54 \%$, eosinophils $3 \%$, band cells $6 \%$, lymphocytes $28 \%$, monocytes $9 \%$, with occasional metamyelocytes). The E.S.R. was $25 \mathrm{~mm}$. in the first hour (Westergren). Examination of a bone marrow biopsy showed no abnormality. Blood urea and plasma electrolytes were normal. Total serum protein was $7 \cdot 4 \mathrm{~g}$. per $100 \mathrm{ml}$., the albumin $4.8 \mathrm{~g}$. per $100 \mathrm{ml}$., the globulin $2 \cdot 6$ g. per $100 \mathrm{ml}$., and there was an increase in the $\alpha 2$ globulin on paper electrophoresis. The antistreptolysin 0 titre was less than 50 units per ml., and culture of an oropharyngeal swab produced a mixed growth of commensals, but included a scanty growth of a Lancefield group A beta-haemolytic streptococcus. The stools were mucoid and blood-stained, but there were no yeasts, parasites, ova, or cysts on microscopy, and culture yielded no pathogenic bacteria. The faecal fat excretion was $3.9 \mathrm{~g}$. in 24 hours.

Sigmoidoscopy to $15 \mathrm{~cm}$. revealed a grossly abnormal rectal and sigmoid mucosa with a finely granular, haemorrhagic pattern, with a few large ulcers with haemorrhagic bases. A biopsy specimen was reported on as follows: 


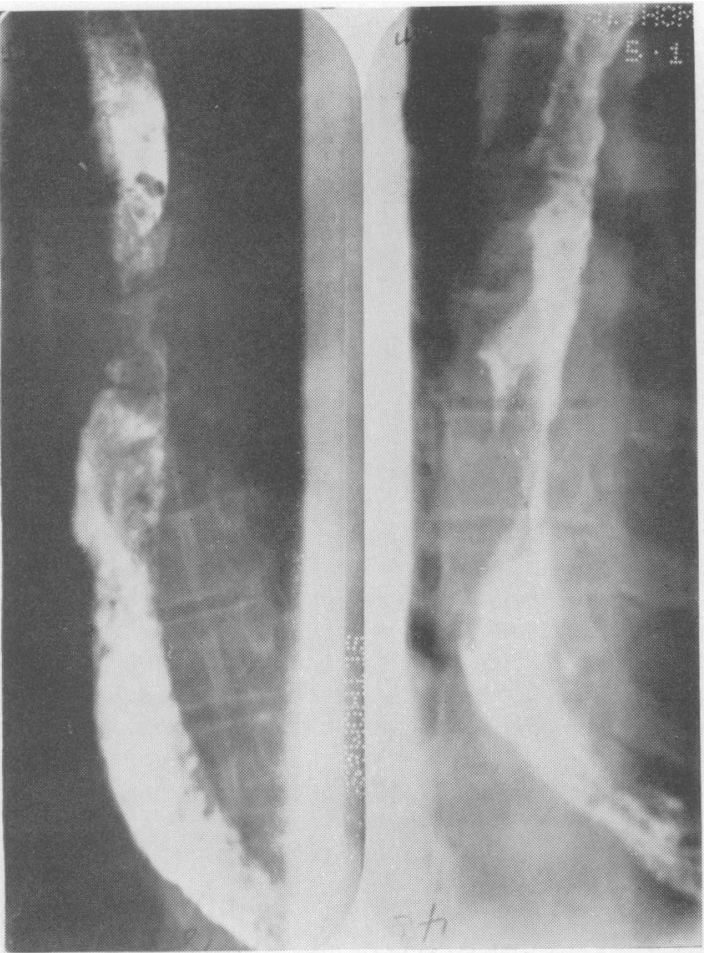

FIG. 1. Barium swallow on 5 January 1965. Gross irregularity of the oesophageal lumen with numerous polypoid filling defects in the barium.

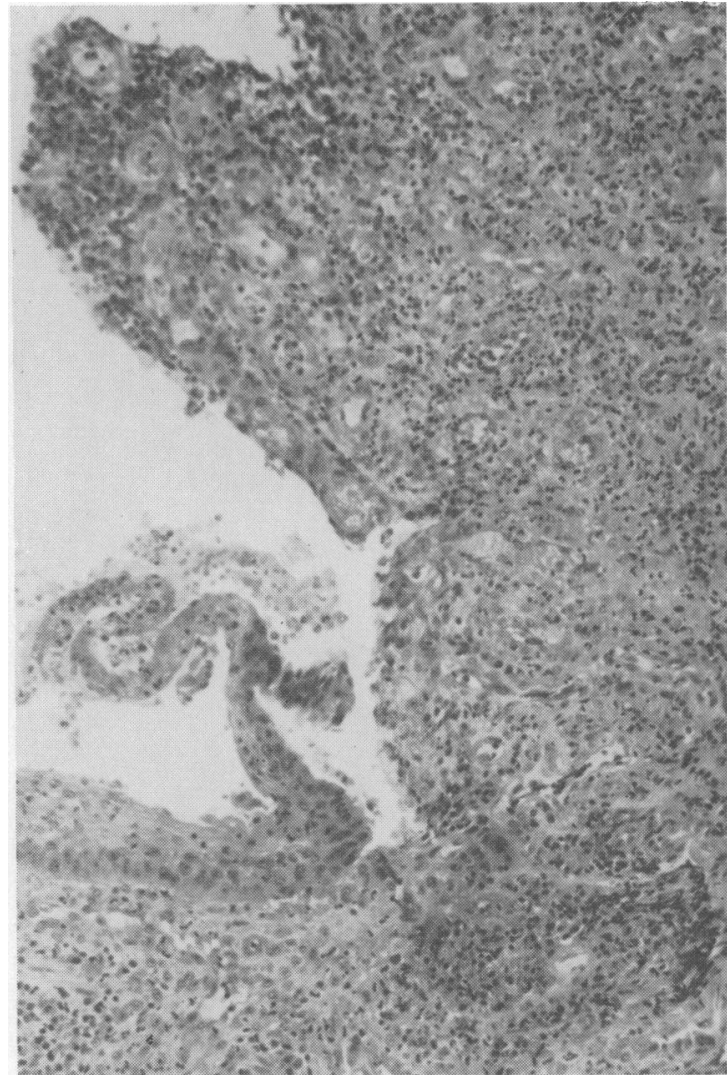

FIG. $2 \mathrm{a}$.

จ

$\vec{\circ}$

$\dot{\overrightarrow{\vec{\omega}}}$

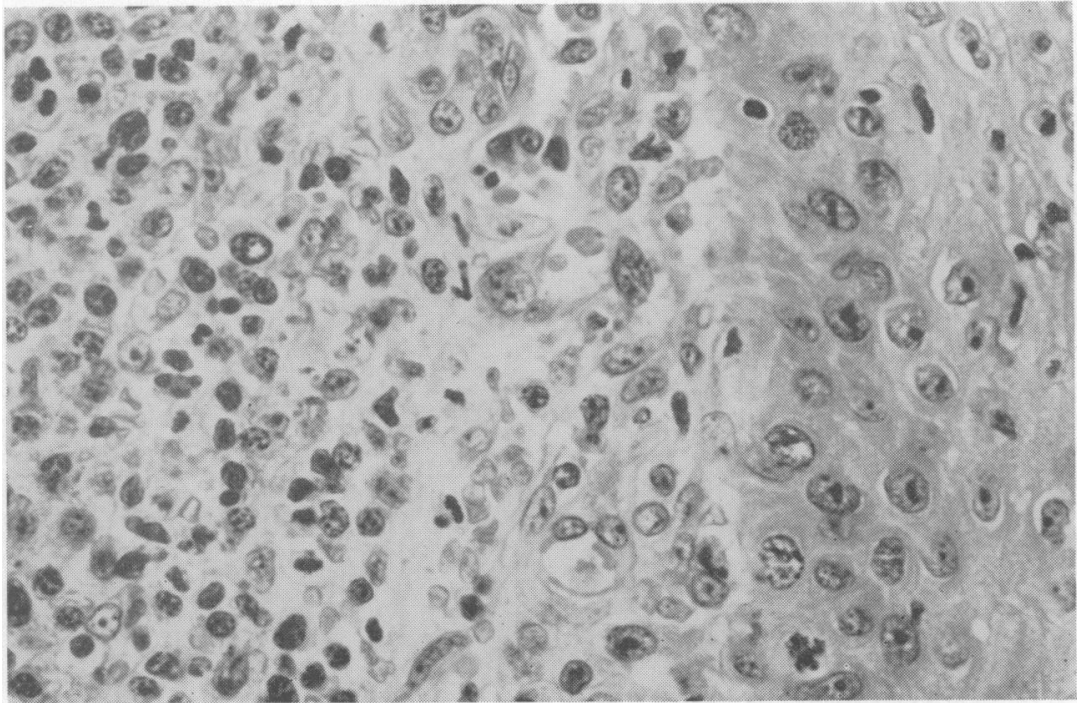

FIG. 2a. Oesophageal ulceration with underlying inflammatory changes. $\times 125$.

FIG. 2b. Chronic inflammatory cell infiltrate. $\times 500$.

FIG. $2 b$. 


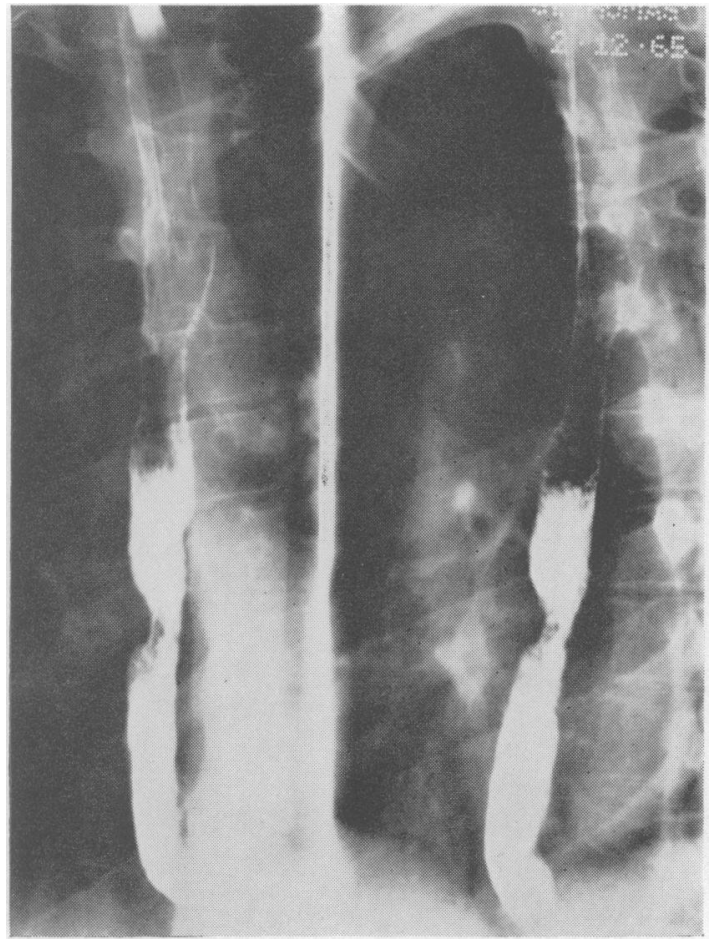

FIG. 3. Barium swallow on 2 December 1966. Minor irregularity persists, and there is now a stricture at the junction of the middle and lower thirds.

'There is severe chronic inflammation of the large bowel mucosa extending into the underlying muscle coat. There are no giant cells or fissures to suggest Crohn's disease. The appearances are consistent with ulcerative colitis.'

Barium enema examination showed considerable ulceration and pseudo-polyp formation from just above the caecum to the descending colon. Barium swallow showed gross irregularity and ulceration in the lower two thirds of the oesophagus (Fig. 1). A small bowel meal was normal.

On 6 January oesophagoscopy was performed by Mr. Kent Harrison, who reported shallow ulceration of the oesophagus, with gross diffuse irregularity and polyp formation. Three biopsies were taken, two from different levels in the oesophagus and one from the pharynx. In all three specimens the oesophageal mucosa was chronically inflamed and oedematous, with a dense infiltrate of plasma cells, lymphocytes, and histiocytes (Fig. 2). In none of the biopsies were specific bacterial or fungal elements seen. Fluid from the oesophagus was aspirated at bronchoscopy, and sent for bacteriological examination. The flora included Gram-positive cocci in pairs and clumps, with pus cells, but culture, including a culture on Sabouraud's medium, grew commensals only, and no Candida albicans. On the basis of the initial report on the barium swallow, which suggested that the oesophagitis might be monilial, and in spite of the negative results of the culture on Sabouraud's medium, the patient was treated with Nystatin lozenges 500,000 units q.i.d. This was followed by a rapid improvement in the dysphagia. The prednisone with which he was initially treated was tailed off, and replaced by prednisolone disodium phosphate (Predsol) $20 \mathrm{mg}$. retention enemas o.d. with considerable improvement in the diarrhoea. The patient was discharged on 30 January 1965 and was symptom free on all occasions in the follow-up clinic. A repeat barium swallow in December 1965 showed numerous irregularities and polypoid filling defects with a constant narrowing of the oesophagus, approximately $10-12 \mathrm{~cm}$. above the diaphragm (Fig. 3). Oesophagoscopy showed multiple polyps and papilliferous lesions throughout the oesophagus, with the mucous membrane covering these lesions, and between them, apparently normal. Biopsy of one of these polyps showed chronic inflammatory changes, the stroma showing vascular proliferation with surrounding chronic inflammatory cell infiltrate consisting of plasma cells and lymphocytes. The overlying squamous epithelium was hyperplastic. The sigmoidoscopic appearances at this time showed a uniformly granular and haemorrhagic mucosa, compatible with mild ulcerative colitis.

The patient remains well and symptom free, and the Predsol retention enemas are being tailed off. The mild iron-deficiency anaemia has been successfully treated with oral iron preparations.

\section{DISCUSSION}

The question of whether the association of ulcerative lesions at either end of the gastrointestinal tract is fortuitous, or whether they are manifestations of the same disease process is one that cannot be answered conclusively. Review of the extensive literature would indicate that the association is very rare. In a study of 60 cases of benign stricture of the oesophagus superimposed on oesophagitis, Benedict and Sweet (1948) include two with ulcerative colitis, and in a necropsy series of 82 cases of acute ulcerative oesophagitis studied by Bartels (1935) six had chronic ulcerative colitis. These figures would suggest that the association is more than coincidental. On the other hand, most of the patients in the latter series were severely debilitated and five of the six had had abdominal operations, which are well known as a cause of oesophagitis, especially if there has been vomiting or nasogastric intubation (Butt and Vinson, 1936).

None of the other alimentary system lesions known to be associated with oesophagitis (Benedict and Sweet, 1948), namely, hiatus hernia, duodenal ulcer, and chronic cholecystitis, were present in our patient, nor had there been vomiting or nasogastric intubation. There was no history of alcoholism, and though some degree of iron-deficiency anaemia was present, the radiological and histological appearances of the oesophagus were not those of a sidero- 
paenic type of oesophagitis (Waldenström and Kjellborg, 1939; Wright, 1965). The radiological appearances were very similar to those described by Grieve (1964) in monilial oesophagitis; however, Candida albicans was neither recovered from oesophageal aspirates nor seen in the oesophageal biopsy specimens.

Thus it would seem likely, though by no means certain, that the colonic and oesophageal lesions are manifestations of the same disease process. The question of whether ulcerative colitis is a generalized disease, or whether the remote 'complications' are a consequence of the colitis, is at present a speculative one (Edwards and Truelove, 1964). Whatever the answer, it appears that no part of the alimentary tract is exempt from involvement, whether it be stomach (Salem, Truelove, and Richards, 1964; Taylor, $1965 \mathrm{a}$ and b; Wright and Truelove, 1966), small bowel (Salem, Truelove, and Richards, 1964), or oesophagus.

We are grateful to Professor W. I. Cranston, Dr. B. Creamer, and Dr. J. R. Tighe for their help in the preparation of this paper.

\section{REFERENCES}

Bartels, E. C. (1935). Acute ulcerative esophagitis. A pathologic and clinical study of 82 cases observed at necropsy. Arch. Path., 20, 369-378.

Benedict, E. B., and Sweet, R. H. (1948). Benign stricture of the esophagus, with special reference to esophagitis, hiatus hernia, esophageal ulcer and duodenal ulcer. Gastroenterology, 11, 618-628.

Berdal, P., and Harnaes, K. (1956). Ulcerative colitis with coexistent ulcerative oesophagitis. (In Norwegian.) Nord. Med., 55, 511-513.

Butt, H. R., and Vinson, P. P. (1936). Esophagitis. II. A pathologic and clinical study. Arch. Otolaryng., 23, 550-572.

Edwards, F. C., and Truelove, S. C. (1964). The course and prognosis of ulcerative colitis. Part III. Complications. Gut, 5, 1-15.

Grieve, N. W. T. (1964). Monilial oesophagitis. Brit. J. Radiol., 37, 551-554.

Salem, S. N., Truelove, S. C., and Richards, W. C. D. (1964). Smallintestinal and gastric changes in ulcerative colitis: a biopsy study. Brit. med. J., 1, 394-398.

Taylor, K. B. (1965a). Role of immune responses in the gastrointestinal tract. Fed. Proc., 24, 23-28.

- (1965b). Immune mechanisms in gastroenterology. In Recent Advances in Gastroenterology, edited by J. Badenoch and B. N. Brooke, pp. 24-48. Churchill, London.

Waldenström, J., and Kjellberg, S. R. (1939). The roentgenological diagnosis of sideropenic dysphagia. Acta radiol. (Stockh.), 20, 618-638.

Wright, R. (1965). Sideropenic dysphagia in a patient with ulcerative colitis. Amer. J. Med., 38, 274-278.

- , and Truelove, S. C. (1966). Auto-immune reactions in ulcerative colitis. Gut, 7, 32-40. 